\title{
DETECTION OF MEDICAL INFORMATION USING LSTM
}

\author{
Gi Sung Lee ${ }^{1}$ and Jong Chan Lee ${ }^{2 *}$ \\ ${ }^{1}$ Division of Computer \& Game, Howon University, Korea \\ ${ }^{2}$ Dep. of Computer Information Engineering, Kunsan National University, Korea \\ ${ }^{1}$ ygslee@howon.ac.kr, ${ }^{2}$ chan2000@kunsan.ac.kr
}

Abstract - The growing number of medical consumers aiming for a better quality of life is boosting the Online to Offline (O2O) medical marketing industry, which saves time and money of clients by choosing reliable medical facilities and receiving high-quality medical services based on medical information from blogs distributed on the Web. Unstructured data generated from the Internet, mobile, SNS, etc., cannot guarantee the credibility of medical information because it directly or indirectly reflects the interest, preference, and expectation of the author in addition to professional medical knowledge. In this study, we propose a blog detection system that provides users with a higher quality medical information service by detecting medical blogs using big data and Long Short-Term Memory (LSTM) processing. We collect and analyze various medical blogs on the Internet based on the big data and machine learning technology, and develop a personalized health recommendation system for each disease. It is expected that the users will be able to maintain their health condition by continuously checking their health problems and taking the most appropriate measures. blog

Keywords - Big data, Long Short-Term Memory, TF-IDF, Doc2Vec, Medical

\section{INTRODUCTION}

With the increase in medical consumption aiming at the quality of life in a modern society, interest in the wellness industry providing customized healthcare services is continuously expanding. As a result, attempts to aggregate and analyze health care blogs to provide personalized health care information are growing increasingly in order to provide the best results for individuals at a relatively low cost and more easily by applying ICT convergence and composite technologies [1]-[2].

Under the expansion of the wellness industry, users can utilize a similar disease community to share information with similar diseases, and realize personalized health management by Q \& A service through specialists. With the development of smart devices, however, many people are getting major medical information from Internet blogs, but the fierce competition among peers has led to a flood of promotional articles aimed at raising the number of views. Publicity blogs are characterized by out-of-the-box celebrity theft, ambiguous sentence closures, inconsistencies in title and content, and propaganda of a particular object or method. Not only medical articles, the most important feature of medical blogs, but also advertisements that attract readers' attention are frequently produced [3]-[4].

Received: May 10, 2019

Reviewed: August 12, 2019

Accepted: September 2, 2019

* Corresponding Author 
Therefore, there is a problem that the user has to repeatedly search for obtaining medical information of interest, and the reliability of the publisher of the medical information blog is poor. In addition, unstructured text data on medical blogs is directly or indirectly reflected advertising of medical products or the promotion of medical devices in addition to factual knowledge, which makes it difficult to ensure the reliability of medical information. Furthermore, due to the various characteristics of language, there are difficulties in analyzing and deriving its meaning. Specifically, the problems in development are described as follows.

- Poor taxonomy of the database to store collected text data makes it difficult to accommodate Web data with large capacity, unstructured and real-time characteristics.

- Various similar medical data such as medical care, food, exercise, culture and product information are generated, but it is difficult to process them in batches or in real time.

- Because existing medical information services have limited scale of actual assessment by managing data individually, there is a lack of evidence to objectively determine the accuracy of medical information.

- A growing number of blogs are posting similar ads under the pretext of medical information. The content has become increasingly sophisticated, making it difficult to exclude advertising blogs as a general search method.

In this study, we propose a blog detection system to collect domestic health information blogs existing on the Internet and to extract the characteristics of medical and advertising articles by making them big data, and to classify medical blogs and public relations blogs based on them. This is a personalized health information service that distinguishes it from existing services and classifies publishers through the blog detection system (professional caregivers, laypersons, and advertisers) to provide more accurate, high-quality medical information services to users.

\section{BLOG DETECTION SYSTEM}

\subsection{SYSTEM STRUCTURE}

Big data systems collect data from healthcare-related blogs through dedicated web crawlers, classify data, and perform processing and analysis. From these analyzed results, the proposed system provides users with high-quality service by simultaneously providing customized health care-related and institutional blogging information. In this study, a big data distributed processing system is established to meet the above objectives. Figure 1 is a configuration diagram of Hadoop-based big data systems [5]. 


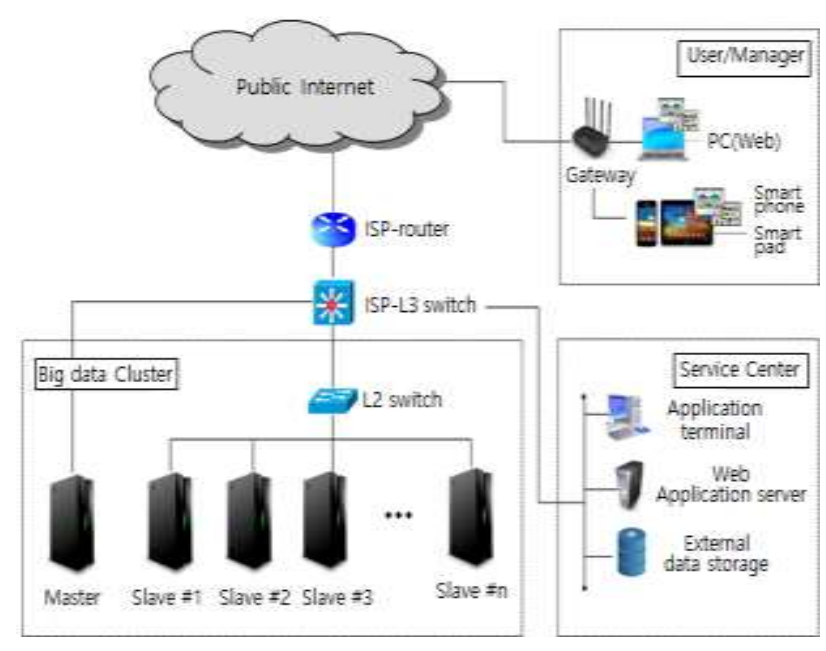

Fig. 1 Structure of Big Data System

The system used four servers to establish a distributed processing cluster by setting one of them as the master server and the other three as the slave server. For the high availability of the big data processing system, the slave \#1 server is built to perform its work on behalf of the master server in the event of a master server failure, and the system is deployed so that the remaining slave servers could take its place even if up to two slave servers fail at the same time. Each slave server is connected to the L2 switch to communicate with each other, the master server is placed on the L3 switch for cluster-tocluster connectivity, and the servers needed for control and service are placed on the L3 switch [5].

\subsection{COLLECTING, PROCESSING AND ANALYSIS OF MEDICAL BLOGS}

This study introduce three processing functions as shown in Table I to establish a blog detection system. The crawled data can be categorized, analyzed and processed into optimized information, and then classified into advertising blogs and medical information blogs.

Table I. Collection/Processing/Analysis of a Blog

\begin{tabular}{|c|l|l|}
\hline Steps & \multicolumn{1}{|c|}{ Functions } & \multicolumn{1}{c|}{ Implementation methods } \\
\hline \multirow{4}{*}{$\begin{array}{c}\text { Level1: } \\
\text { Data } \\
\text { collection/ } \\
\text { processing }\end{array}$} & $\begin{array}{c}\text { Selection of blogs } \\
\text { for data collection }\end{array}$ & $\begin{array}{l}\text { - Individual and group websites } \\
\text { - Website of middle and large hospital } \\
\text { - Website of the pharmaceutical company }\end{array}$ \\
\cline { 2 - 3 } & Collection of data & - Data collection through web crawler \\
\cline { 2 - 3 } & Processing of data & $\begin{array}{l}\text { - Natural language processing } \\
\text { - Morphological analysis } \\
\text { - Extraction of keywords using KoNLy, Twitter, etc. }\end{array}$ \\
\hline \multirow{2}{*}{$\begin{array}{c}\text { Level 2: } \\
\text { Data analysis }\end{array}$} & Conversion of data & - Blog filtering by applying TFIDF method \\
\cline { 2 - 3 } & Primary filtering & $\begin{array}{l}\text { - Structured data conversion of unstructured data } \\
\text { - Conversion of unstructured data into document matrix } \\
\text { using Doc2Vec }\end{array}$ \\
\cline { 2 - 3 } & Secondary filtering & - Blog filtering using LSTM \\
\hline $\begin{array}{c}\text { Level 3: } \\
\text { Service } \\
\text { provision }\end{array}$ & Provision of service & $\begin{array}{l}\text { - Providing extracted medical information } \\
\text { - Providing extracted medical facility information }\end{array}$ \\
\hline
\end{tabular}


The overall flow of processing functions and analysis methods in Steps 1 and 2 is as follows.

- The data collection phase collects data from personal and hospital blogs through Web crawlers and stores the data in an analytical form.

- As a pre-processing of data, natural language processing and morphology analysis of stored blog text data is performed. This is the process of removing factors that have an inappropriate effect on blog judgment. Except for Korean, the Chinese and English characters, numbers, and emoticons were filtered for emotional classification. Then, the preprocessing process is performed by dividing them into usage, body language, adverb, and type form elements through a morpheme analyzer.

- Unformatted text data is structured so that it can be entered into a structured MongoDB through a text mining process [6]-[7].

- The selected features constitute a medical information dictionary in ascending order. A medical information dictionary is needed to obtain the unique number of the word when quantifying vectors. Medical information extracted by a morpheme analyzer is encoded so that it can be configured as vectors. Medical information eliminates redundant data and converts to file types sorted in ascending order.

- Learning processing is the creation of a learning model that will be used in the classifier using learning data that is the basis for learning. The most important thing in the process of learning is to distinguish between false learning data and real learning data and to learn these two classes separately.

- Blog categorization is the classification of blogs using a category-specific categorizer model created from the learning process. Vector data of each quality generated from the learning process is entered into the blog classification processing to determine the results of the classification.

2.2.1 COLLECTING AND STORING BLOGS: Web crawling is performed to collect various medical blogs. Web crawling functionality is established and the automatic maintenance functions of Web sites, such as link checking and HTML code validation, as well as the collection of certain forms of information from webpages. 10,000 blogs are collected between May 2013 and January 2019 to collect health carerelated blogs. The collected text data is stored in the MongoDB of the blog detection system and the attributes needed for analysis are stored in JSON format. JSON files are created for each module as shown in Figure 2, and the stored properties consist of the key values of JSON: Module, Subject, and Description.

\{

"General": [

\{"subject": "Diabetes and Symptoms"

"description": "There are two types of diabetes. Type 1 diabetes usually appears in childhood and is sometimes referred to as childhood diabetes. It is a disease that insulin is artificially administered because insulin secretion does not occur in the body. $|r| n|r| n "$ \} ]\}

Fig. 2 Data structure for blog data

In this study, MongoDB [8], which is specialized in storing unstructured data, is used. The structure of data stored in MongoDB through blog data processing is as in Figure 3. 
After dataizing information on medical blogs, the blog detection system categorizes advertising blogs and medical blogs. In Figure 3, medical data are managed by date via the id and classified by disease name in a subsection called myocardial infarction. In the actual data analysis process, myocardial infarction data stored in MongoDB is analyzed through text mining.

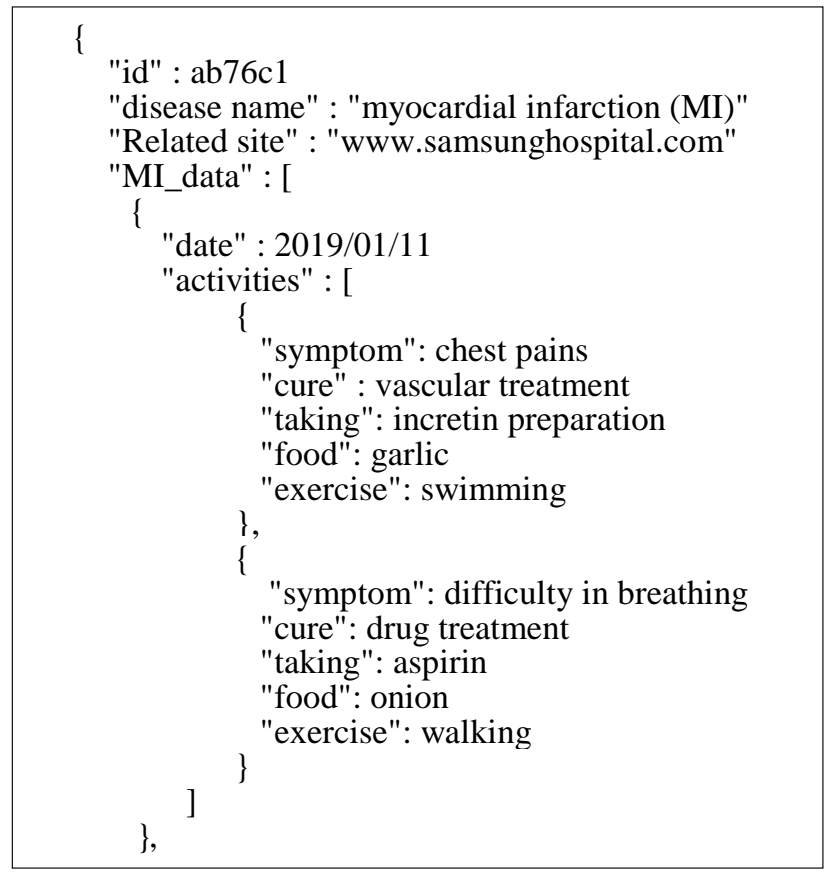

Fig. 3 Structure of data in MongoDB

2.2.2. EXTRACTING BLOG FEATURES: In MongoDB, fifteen files of each module are saved in JSON format (modules, subject, description). The processing of a string is first performed by applying auto-spaced writing, assuming that the registrar does not generally take into account space writing in registering the report. The properties are then stored in the MongDB in JSON format and divided into 15 files per module for module, subject, and description information.

In the case of Han-gul, KoNLPy's Komoran morpheme analyzer [9] is used to convert all morphemes to lower case letters and remove punctuation, spaces, and numbers. In addition, natural language processing is performed on each word by removing function words such as articles, prepositions, postpositions, and conjunctions, which have insufficient roles in expressing sentences. In addition, stemming, which extracts the root or removes the affixation, enhances the efficiency of text processing by treating words of the same meaning as a single word [10].

In the blog detection system, text mining is applied to read advertising blogs and medical blogs. The parameters for extracting characteristics of an advertising blog are as follows.

- The Number of words in the title and body of the Blog: Advertising blogs consist mostly of fewer letters than regular medical blogs. Therefore, it is used as a feature to distinguish advertising blogs by comparing the number of words in the body.

- The frequency of use of certain hospitals, medicines, dry foods, health drinks, etc. : This is also one of the main features, as the advertising blog mainly promotes the treatment of diseases in certain hospitals, certain drugs in pharmaceutical companies, and food and beverages in health food companies. 
- Correspondence between the subject and the body: In most advertising blogs, the degree of agreement between the topic sentence and the body is low.

- Use of simple exclamation: The use of specific words at the end of the text (exclamation mark, question mark, etc.) is also an important feature.

- Overuse of positive and negative patterns: The use of extreme positives or negatives to promote a particular content is also a major feature.

The parameters for medical blog detection are as follows.

- Detection of medical jargon by disease: If a particular disease is detected in the text of a blog or if a disease is classified as treatable, the blog is detected.

- Detection of disease-specific symptoms: Whether it is a medical blog or not is detected based on symptoms of the disease.

\subsection{CLASSIFICATION OF MEDICAL AND ADVERTISING BLOGS}

2.3.1. CLASSIFICATION OF BLOGS USING TF-IDF: In order to store the text data in a meaningful numerical value on the basis of the medical blog converted into the word-disease matrix, the weight for each word is calculated using a term frequencyinverted document frequency (TF-IDF) [11]. In order to store the text data in a meaningful numerical value on the basis of the medical blog converted into the worddisease matrix, the weight for each word is calculated using a term frequency-inverted document frequency.

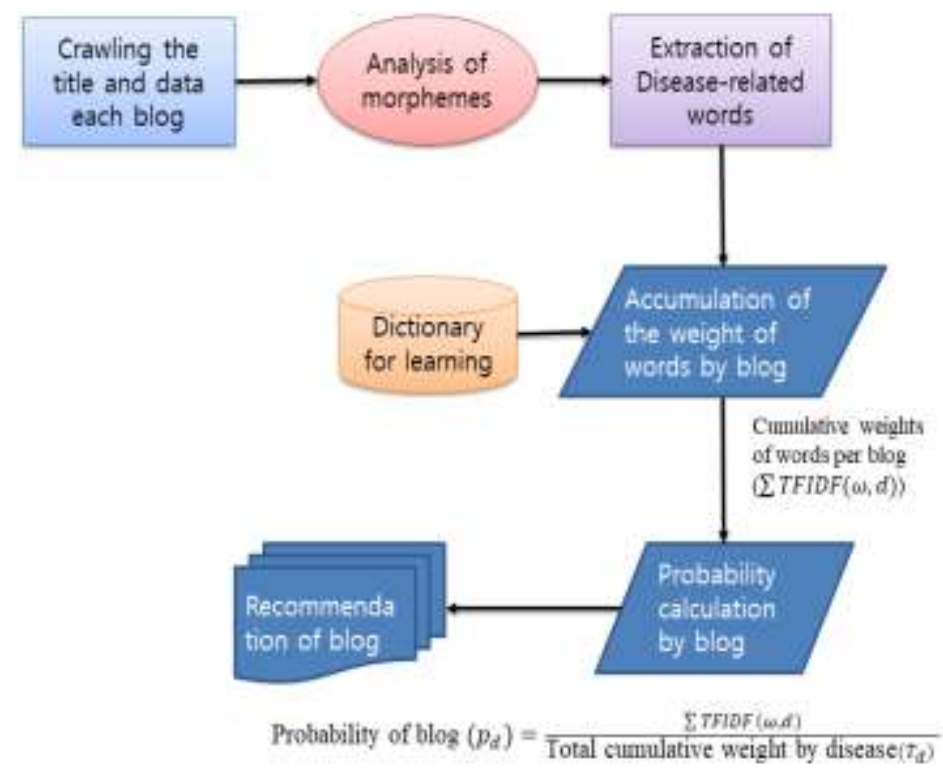

Fig. 4 Data processing structure

We divide weighted words into nouns and derive variables to identify the constituents of medical data through frequency analysis and ratio analysis. As shown in Figure 5, Long Short-Term Memory (LSTM) is used for element analysis. The LSTM derives the components of medical data, and based on them, determines the determining conditions for the type of disease. TF-IDF is used to perform analysis at the sphere and clause level. An associated query map is established for searching the similar blog. When using the TF-IDF method to determine the importance of the associated search term, words with the greatest weighting value are adopted as keywords in the document. 


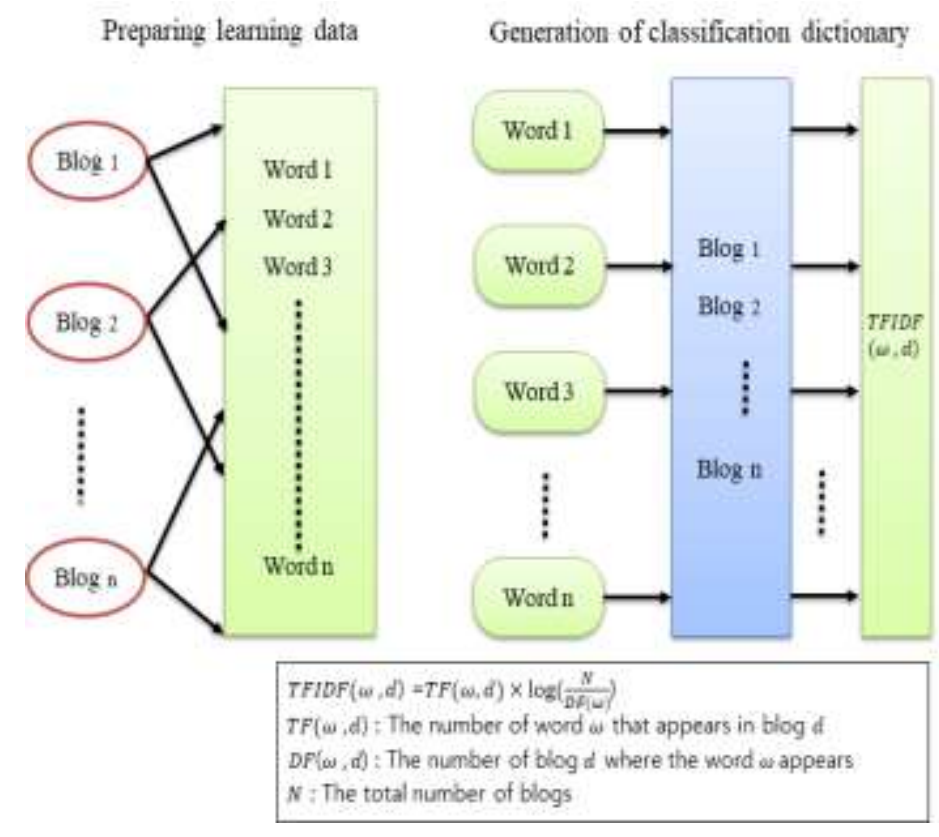

Fig. 5 Structure of TF-IDF

2.3.2. CONVERSION OF DOCUMENTS TO VECTOR USING Doc2Vec [12]: The machine learning model used in this study requires that the text string cannot be entered as it is for training and that the input value is expressed numerically in a fixed length so that the computer can recognize it. Therefore, a pre-processing process is needed to express a document or the words contained in the document in a vector of a fixed size. Using gensim's Doc2vec library, documents and words are converted to vectors. Gensim's Doc2vec library takes a document's token as input and generates a vector of the document and the words contained in it. The vector size of each document and word is set to 300 dimensions, and the parameter value of the constructor of the Doc2vec class is as shown in Table II.

Table II. Parameters in Doc2Vec

\begin{tabular}{|c|l|c|}
\hline Parameter & \multicolumn{1}{|c|}{ Explanation } & Set value \\
\hline alpha & Initial value of learning rate & 0.025 \\
\hline min_count & Ignore words whose frequency is less than the set value & 1 \\
\hline window & Maximum distance between current and predicted words & 10 \\
\hline size & Dimension of output vector & 300 \\
\hline sample & $\begin{array}{l}\text { threshold value for stochastic exclusion of frequently } \\
\text { appearing words }\end{array}$ & $1 \mathrm{e}-5$ \\
\hline negative & $\begin{array}{l}\text { To reduce softmax computation of the neural network output } \\
\text { layer, the number of words selected as the target class among } \\
\text { the remaining words, not the predictive word. }\end{array}$ & 15 \\
\hline workers & The number of workerthreads to be used to train the model. & 10 \\
\hline
\end{tabular}

2.3.3. CLASSIFICATION OF BLOGS WITH LSTM: Blog data filtered from Doc2Vec filter is used as input value for LSTM [13]-[14]. LSTM is a kind of recurrent neural networks that recognizes patterns in data in the form of letters, genes, hand letters, voice signals, sensor-detected data, stock price, etc., and it is possible to predict words with high accuracy by learning the dependencies between words in the time series data. LSTM cannot use a document as an input value, so data should be converted into vectors 
that can represent numbers. In addition, since text data is different in length from image data, it must be converted into a fixed length vector.

- Morpheme analysis files of disease data: A model is created by learning diseaserelated symptoms, medical subjects, and disease classifications, and using the model to classify new blogs. Blogs are categorized using the Doc2Vec filter and used as additional learning data.

- Vector conversion of medical information: For the conversion of text data to vector data, each word is given an ID and a vector is created based on the frequency of appearance and the order of alignment of the IDs. Here, we ignored the order of the words, and used only their frequency of appearance. It also performs the numericalization of input values by applying Bag of Words (BoW), which numerically indicates the existence of a word in the text. First, morphological analysis of the input text is performed and an ID is assigned to each word. In addition, the number of occurrences of the word is obtained. Thus, the sentence can be expressed based on the number of occurrences of the word.

- Classification of Medical blogs: The process of classifying text using LSTM is as follows.

(1) Eliminate unnecessary adverbs from text data

(2) Translate words into numbers based on a medical dictionary using TF-IDF

(3) Calculate the rate of appearance of medical words within a file using Doc2Vec

(4) Learn data using LTSM

(5) Enter test data and check success rate

In the LSTM model, the input layer is also represented in a $500 \times 300$ dimensional vector matrix using the Doc2 Vec library. Texts on a blog converted to vectors are sequentially entered into hidden layers to be learned. The hidden layer takes into account both the previous state value and the current input in determining the state value of each step. Previous output values are also reflected in the learning and classification of current inputs. In Table III, the number of units in LSTM cells is set to 500, which is set to the level of the output layer. The output layer is classified into 15 modules by applying Softmax.

Table III. Parameters in LSTM

\begin{tabular}{|l|l|}
\hline \multicolumn{1}{|c|}{ Parameter } & \multicolumn{1}{c|}{ Set Value } \\
\hline Max Sentence Length & 500 \\
\hline Unit Number & $500(200 \sim 500)$ \\
\hline Forget bias & 1.0 \\
\hline Dropout Rate & 0.5 \\
\hline Learning Rate & 0.001 \\
\hline Optimizer & Adam \\
\hline Doc2Vec & 300 \\
\hline Classification / Softmax & 15 \\
\hline
\end{tabular}

\section{PERFORMANCE EVALUATION}

In this study, the medical keyword dictionary for each disease is established as shown in Table 4 [15]-[20]. The data crawled from the medical site is separated into sentences, and each sentence is converted into a token array after the morphological analysis. The converted token is stored in the medical keyword dictionary. This medical dictionary is used as a criterion of importance in TF-IDF, as a filtering condition in Doc2Vec, and as an input value and learning parameter in LSTM. 
Table IV. A Medical Dictionary by Disease

\begin{tabular}{|l|l|}
\hline \multicolumn{1}{|c|}{ Medical dictionary } & \multicolumn{1}{c|}{ Disease related medical word } \\
\hline Diabetes & blood sugar, insulin, hyperglycemia \\
\hline High blood pressure & hypertension, diastole, atherosclerosis \\
\hline Obesity & Overweight, body/abdominal obesity \\
\hline Hyperlipidemia & dyslipidemia, cholesterol, High bleeding \\
\hline Atherosclerosis & heart disease, cerebrovascular disease \\
\hline Angina pectoris & heart disease, ischemia, cardiomyopathy \\
\hline Stroke & paralysis, ischemia, ischemic attack \\
\hline Lung disease & pneumonia, pulmonary tuberculosis, asthma \\
\hline Liver disease & sarcoidosis, fatty liver, liver cancer \\
\hline Gastric disease & gastritis, gastric ulcer, gastric cancer \\
\hline Joint disease & joints, arthritis, degenerative, rheumatic \\
\hline Osteoporosis & skeletal system, fracture, bone density \\
\hline thyroid & thyroid cancer, thyroiditis \\
\hline headache & migraine, tension headache, temporal arteritis \\
\hline Urinary disease & urinary incontinence, prostate, prostatitis \\
\hline Heart disease & cardiovascular, cardiovascular, arrhythmia \\
\hline Eye diseases & conjunctivitis, cataract, glaucoma \\
\hline Skin disease & dermatology, atopy, atopic dermatitis \\
\hline Depression & depressive disorder, sleep depression \\
\hline Stress & adaptive disorder, anxiety disorder \\
\hline Gastric cancer & gastric cancer, lymph node, gastroscopy \\
\hline Colon cancer & colon, colon polyps, colitis \\
\hline & \\
\hline
\end{tabular}

Table IV shows the evaluation items and performance targets of the proposed blog detection system. The blog detection system performs TF-IDF, Doc2Vec, and LSTM to evaluate stored blogs in MongoDB, and determines whether the blog is an advertisement blog or a medical blog.

Table V. Test Items and Performance Objectives

\begin{tabular}{|l|l|}
\hline \multicolumn{1}{|c|}{ Test item } & \multicolumn{1}{c|}{ Performance objectives } \\
\hline Detection of advertising blogs & More than $87.3 \%$ \\
\hline Detection of medical blogs & More than $95.7 \%$ \\
\hline Accuracy of Big Data Classification by Disease & More than $96.5 \%$ \\
\hline
\end{tabular}

Through the evaluation of the blogs stored in MongoDB, it is confirmed that more than $87.3 \%$ of actual blogs is detected among those detected as advertisement blogs. In addition, among the blogs detected as medical blogs, more than $95.7 \%$ of the actual medical blogs are included. More than $96.5 \%$ of the topics on the actual blog were matched with information that is tagged by disease.

\section{CONCLUSION}

Health is a major concern for modern people, but the existence of a variety of similar medical information on the Internet makes it difficult to trust and, in addition, it is almost impossible to provide customized medical information suitable for specific users. In this 
study, we proposed a blog detection system to collect a large number of medical related blogs on the Internet through big data Technology, and by using machine learning technology, detect advertising blogs from them, as well as classify them by disease. The proposed system enabled customized health recommendation services to realize information sharing with people with similar diseases and personal-led health care. In the future, it is necessary to study medical information dictionaries and advertisement dictionaries precisely and apply reinforcement learning.

\section{ACKNOWLEDGMENTS}

This work was supported by the Howon University Fund, 2019.

\section{REFERENCES}

[1] R. M. Abbas, N. Carroll and I. Richardson, "In Technology We Trust: Extending TAM from a Healthcare Technology Perspective." International Conference on Healthcare Informatics, New York, USA, 348-249, June 2018.

[2] R. J. Conejar and H. K. Kim, "Proposed Architecture for U-Healthcare Systems." International Journal of Software Engineering and Its Applications 9.7 (2015): 213-218.

[3] Y. E. Gelogo and H. K. Kim, "Integration of Mobile Computing to Ubiquitous Healthcare." International Journal of Software Engineering and Its Applications 9.9 (2015): 295-302.

[4] S. K. Ki, M. S. Lee, J. K. Paik, "Perspective of Ubiquitous Healthcare System for Elderly People." Journal of Korean Society of Sport Policy 13.2 (2015): 87-98.

[5] E. Ferrara and P. Meo, et al. Web Data Extraction, Applications and Techniques - A Survey: ELSEVIER, 2014.

[6] P. Mohata and S. Dhande, "Web Data Mining Techniques and Implementation for Handling Big Data." International Journal of Computer Science and Mobile Computing 4.4 (2015): 330-334.

[7] B. Dayley. Node.js, MongoDB, and AngularJS Web Development: Addison-Wesley, 2014.

[8] B. Singh and S. Kumar, "An Effective Information Retrieval with Keyword Optimization." International Journal of Computer Technology and Applications 5.1 (2014): 174-177.

[9] M. K. Kim, N. K. Kim, and I. H. Jung, "Including Automatic Keyword Extraction Techniques of Shopping Through Internet Search History Analysis." Journal of Intelligent Information Systems (2014): 123-136.

[10] J. C. Lee and K. J. Park, "Design of Food Management System Using NFC Tag." Journal of Korea Society of Computer and information 23.5 (2018): 25-29.

[11] A. Guo and T. Yang, "Research and improvement of feature words weight based on TFIDF algorithm." International Conference on Information Technology, Networking, Electronic and Automation Control, Chongqing, China, 415-419 May 2016.

[12] A. Guo and T. Yang, "Sentiment analysis on Twitter data with semi-supervised Doc2Vec." International Conference on Computer Science and Engineering, Antalya, Turkey, 1-5 October 2016.

[13] R. Nishimura, M. Higaki and N. Kitaoka, "Mapping Acoustic Vector Space and Document Vector Space by RNN-LSTM." Global Conference on Consumer Electronics, Nara, Japan, 329-330 October 2018.

[14] T. M. Breuel, "High Performance Text Recognition Using a Hybrid Convolutional-LSTM Implementation, "International Conference on Document Analysis and Recognition, Kyoto, Japan, 1116 November 2017.

[15] G. S. Lee and J. C. Lee, "A Classification of Medical and Advertising Blogs Using Machine Learning." Journal of Korea Academia-Industrial cooperation Society 19.11 (2018): 730-737.

[16] D. H. Shin and C. B. Kim, "Short-Term Photovoltaic Power Generation Forecasting by Input-Output Structure of Weather Forecast Using Deep Learning." International Journal of Software Engineering and Its Applications 12.4 (2018): 19-24.

[17] M. J. Seo and M. H. Kim, "Data Leakage Detection System based on Deep Learning." International Journal of Reliable Information and Assurance 4.2 (2016): 13-18.

[18] P. Chandra, "A Survey on Deep Learning its Architecture and Various Applications." Asia-Pacific Journal of Neural Networks and Its Applications 1.2 (2017): 7-14.

[19] D. Wang and T. Whangbo, "An Automatic Diagnostic Algorithm for Parkinson's Disease Based on Deep Learning." Asia-Pacific Journal of Neural Networks and Its Applications 2.2 (2018): 13-18.

[20] I. S. Weon and S. G, Lee, "Velocity-based object detection in dynamic environment using YOLO-based deep learning algorithm." International Journal of Multimedia and Ubiquitous Engineering 14.1 (2019): 7-12. 